\title{
Splenic Infarction Associated with Acute Brucellosis: A Case Report ${ }^{*}$
}

\author{
Wissem Hachfi, Foued Bellazreg, Amira Atig, Nadia Ben Lasfar, Naoufel Kaabia, Amel Letaief
}

Department of Internal Medicine and Infectious Diseases, Farhat Hached Hospital, Sousse, Tunisia.

Email: hachfi_wissem@hotmail.com

Received April 19 $9^{\text {th }}, 2012$; revised May 21 ${ }^{\text {st }}, 2012$; accepted June $25^{\text {th }}, 2012$

\begin{abstract}
We report a case of a 21-year-old shepherd who presented with fever, left hypochondrium pain and splenomegaly. Blood tests showed thrombocytopenia and high liver enzymes. Computed tomography scan revealed the presence of hypodense lesions suggestive of splenic infarction. Echocardiography was without abnormalities. Laboratory evaluation for thrombophilia was negative. A bone marrow aspiration and a bone marrow biopsy were normal. Brucella serology was positive. The patient was treated with doxycycline, rifampicin and streptomycin for 12 weeks. Fever, abdominal pain and thrombocytopenia resolved promptly. At one year of follow-up, he remained well and no splenic infarction could be detected on ultrasonography.
\end{abstract}

Keywords: Brucellosis; Splenic Infarction; Thrombocytopenia

\section{Introduction}

Brucellosis, a bacterial zoonose transmitted to humans from infected animals, constitutes a major health problem in many parts of the world, particularly in the Mediterranean basin and the Middle East [1]. Vascular complications have rarely been associated with this disease $[2,3]$. We report herein a case of splenic infarction associated with acute brucellosis.

\section{Patient and Case Report}

A 21-year-old shepherd, who had a history of raw milk consumption, presented to our hospital with a two-week history of fever, night sweats and left hypochondrium pain. Physical examination showed a body temperature of $39^{\circ} \mathrm{C}$ with a tender splenomegaly $3 \mathrm{~cm}$ below the costal margin. Vital signs were normal and no heart murmur was noted. There was no bruising.

Laboratory tests revealed thrombocytopenia $(31,000 /$ $\mathrm{mm}^{3}$ ) and high liver enzymes (AST, $134 \mathrm{U} / \mathrm{L}$; ALT, 105 U/L). White blood cells, haemoglobin, creatinin, fibrinemia and activated partial thromboplastin time were in normal range. Blood cultures were negative. Bone marrow aspiration revealed normal cellular distribution and maturation. Bone marrow biopsy was normal. Abdominal Computed Tomography (CT) scan revealed the presence of various hypodense lesions of triangular morphology without uptake of intravenous contrast (Figure

*The authors report no competing interests.
1), suggesting splenic infarction. Transoesophageal echocardiography (TEE) didn't show lesions of the valves or intracavitary thrombus. Anti-thrombin III, proteins $\mathrm{C}$ and $\mathrm{S}$ were in normal range and antiphospholipid antibodies were negative. Bleeding time was not measured. AntiBrucella agglutination test was positive at a titer of 1/1280. Serological tests for Epstein-Barr Virus (EBV), cytomegalovirus (CMV), Coxiella burnetti and Rickettsia conorii were negative.

The patient was treated with rifampicin $(900 \mathrm{mg} /$ day $)$ and doxycycline $(200 \mathrm{mg} /$ day $)$, associated with streptomycin (1 g/day) during the first two weeks. Fever and abdominal pain resolved within two days and thrombocytopenia resolved within 6 weeks. Serological tests for Brucella performed in five goats and sheep with which the patient had direct occupational contact were all positive. At the end of antibiotic treatment, which was maintained for 12 weeks, no splenic infarction could be detected on ultrasonography and the titer of the agglutination test for Brucella decreased to 1/320. Abdominal CT scan was not performed. At one year of follow-up, the patient remained completely asymptomatic.

\section{Discussion}

In addition to Infective Endocarditis (IE), the only reported cases of splenic infarction coinciding with an infectious disease were associated with the presence of antiphospholipid antibodies [4,5]. 


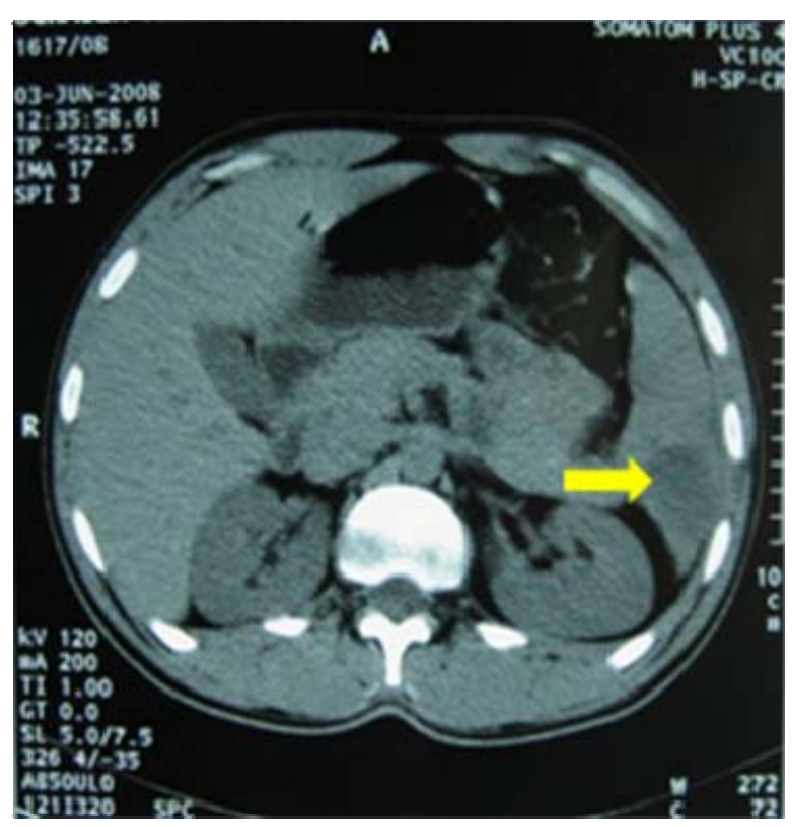

(a)

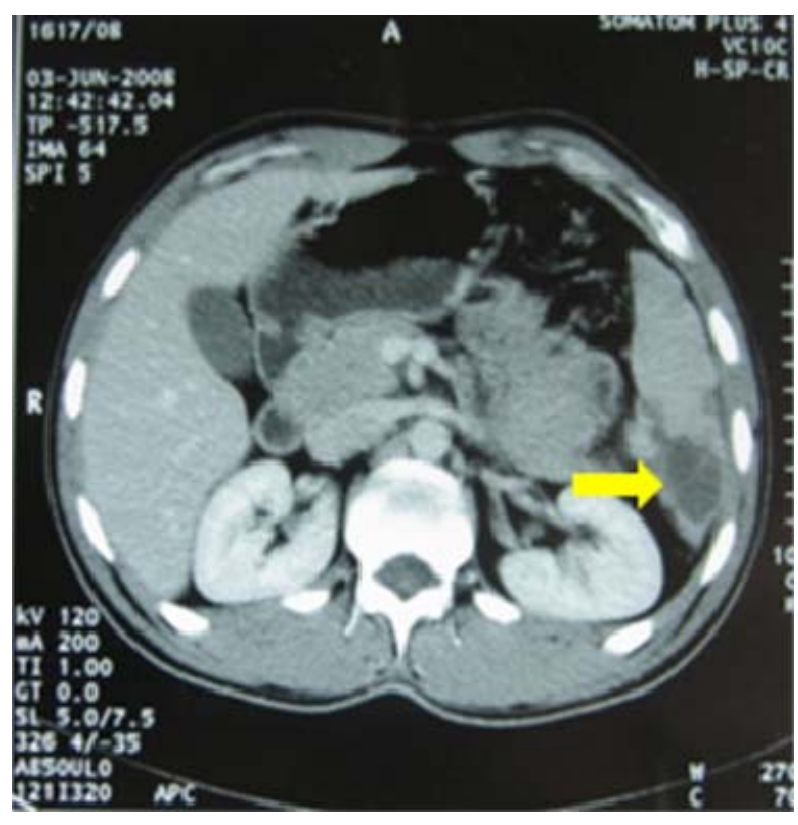

(b)

Figure 1. Abdominal CT scan showing splenic infarction: hypodense splenic lesion (a) without uptake of intravenous contrast (b).

In our case, IE which was the first diagnostic hypothesis was promptly excluded by TEE and laboratory evaluation for thrombophilia was negative. Brucella serology was performed as the patient presented with prolonged fever and had risk factors for brucellosis (occupational contact with goats and sheep in an endemic country). Blood cultures were negative probably because they were not performed on special media since the microbiologist was not initially informed of the suspicion of brucellosis.

Splenic infarction is extremely rare in brucellosis. To the best of our knowledge, only two cases of splenic infarction associated with brucellosis have been reported $[6,7]$.

The first case was observed in a 39-year-old Spanish husbandry that presented with a 7 day history of fever and left hypochondrium pain. Laboratory data showed mild thrombopenia $\left(90,000 / \mathrm{mm}^{3}\right)$ and elevated liver enzymes (ALT UI/L, AST 72 UI/L). Abdominal CT scan showed splenic hypodense lesions without uptake of contrast. A bloody culture was positive for Brucella melitensis and the test agglutination was positive at a titer of 1/1320. An echocardiogram was normal and no thrombosis tendency was observed. The patient was treated with doxycycline and streptomycin. The outcome was favorable.

The second case was observed in an Italian child who presented with fever and left upper quadrant pain. Abdominal CT scan showed splenic triangular, hypodense lesion. Serum and culture exams confirmed the diagnosis of brucellosis. The patient recovered after 2 and halfmonths antibiotic therapy.

The symptoms of splenic infarction are non specific and may include pain in the left hypochondrium, nausea and fever. Contrast CT scan is the best non-invasive test to diagnose splenic infarction, the visualization of a low density lesion without uptake of intravenous contrast is highly suggestive of this diagnosis. Ultrasonography which has a low diagnostic yield can be useful in follow up of patients to detect complications [8].

Physiopathological mechanisms proposed to explain the thrombotic complications in infections include septic emboli associated with IE and endothelial lesions due to local germ infiltration which would favor hypercoagulability [6]. In our patient, vasculitis due to direct endothelial damage by Brucella might be the most plausible explanation.

No data exist that define the indication, duration, or type of treatment of splenic infarction complicating infections. Anticoagulants should be used in the presence of antiphospholipid antibodies [4]. Our patient responded favourably to antibiotics (doxycycline, rifampicin and streptomycin) at standard doses for 12 weeks.

Our case report was also characterized by a persistent thrombocytopenia. This feature is noted in $12.5 \%$ to $26 \%$ of cases of brucellosis [9]. Its mechanisms include hypersplenism, bone marrow suppression due to septicemia, direct damage to platelets by bacteria, hemophagocytosis, granulomatous lesions of the bone marrow or Disseminated Intravascular Coagulation (DIC) [1]. Thrombocy topenia 
associated with brucellosis is generally mild and resolves gradually with antimicrobial treatment [10]. A short-term corticosteroid treatment might be an additional treatment [1]. Intravenous immunoglobulins should be kept for patients with severe, life-threatening hemorrhage [10].

In our patient, the examination of bone marrow aspiration showed normal cellular distribution and maturation, bone marrow biopsy didn't show hemophagocytosis or granulomas and there was no DIC sign. In our patient, appropriate antibacterial therapy is sufficient to restore platelets count within normal range. Therefore, the most plausible mechanisms of thrombocytopenia would be hypersplenism and/or direct platelets damage by bacteria.

\section{Conclusion}

Brucellosis must be considered in patients with prolonged fever and splenic infarction, particularly in patients living in endemic areas.

\section{REFERENCES}

[1] A. Sevinc, N. Buyukberber, C. Camci, S. Buyukberber and T. Karsligil, "Thrombocytopenia in Brucellosis: Case Report and Literature Review," Journal of the National Medical Association, Vol. 97, No. 2, 2005, pp. 290-293.

[2] M. Odeh, N. Pick and A. Oliven, "Deep Venous Thrombosis Associated with Acute Brucellosis: A Case Report," Angiology, Vol. 51, No. 3, 2000, pp. 253-256. doi: $10.1177 / 000331970005100310$

[3] J. Sanchez-Gonzalez, T. Garcia-Delange, F. Martos and J. D. Colmenero, "Thrombosis of the Abdominal Aorta Se- condary to Brucella Spondylitis," Infection, Vol. 24, No. 3, 1996, pp. 261-262. doi:10.1007/BF01781108

[4] M. Capell, T. Simon and M. Tiku, "Splenic Infarction Associated with Anticardiolipin Antibodies in a Patient with Acquired Immunodeficiency Syndrome," Digestive Disease and Sciences, Vol. 38, No. 6, 1993, pp. 1152-1155. doi:10.1007/BF01295735

[5] C. M. Witmer, A. P. Steenhoff, S. S. Shah and L. J. Raffini, "Mycoplasma Pneumoniae, Splenic Infarct, and Transient Antiphospholipid Antibodies: A New Association," Pediatrics, Vol. 119, No. 1, 2007, pp. 292-295.

[6] F. Salgado, M. Grana, V. Ferrer, A. Lara and T. Fuentes, "Splenic Infarction Associated with Acute Brucella Melitensis Infection," European Journal of Clinical Microbiology \& Infectious Diseases, Vol. 21, No. 1, 2002, pp. 63-64. doi:10.1007/s10096-001-0655-3

[7] C. Ruggeri, V. Tulino, T. Foti, L. Carcione, D. Vita, C. Visalli, et al., "Brucellosis and Splenic Infarction: A Case in Pediatric Age," Minerva Pediatrica, Vol. 53, No. 6, 2001, pp. 577-579.

[8] M. Antopolsky, N. Hiller, S. Salameh, B. Goldshtein and R. Stalnikowicz, "Splenic Infarction: 10 Years of Experience," American Journal of Emergency Medicine, Vol. 27, No. 3, 2009, pp. 262-265. doi:10.1016/i.ajem.2008.02.014

[9] İ. Dilek, A. Durmus, M. K. Karahocagil, H. Akdeniz, H. Karsen, A. I. Baran, et al., "Hematological Complications in 787 Cases of Acute Brucellosis in Eastern Turkey," Turkish Journal of Medical Sciences, Vol. 38, No. 5, 2008, pp. 421-424.

[10] M. Özen, Ü. Özgen and S. Güngör, "Management of Brucella-Induced Thrombocytopenic Purpura," The Pediatric Infectious Disease Journal, Vol. 3, No. 3, 2009, pp. 83-85. 